2010-10

\title{
Workspace comparisons of setup configurations for human-robot interaction
}

\section{Zacharias, $\mathrm{F}$}

http://hdl.handle.net/10026.1/15022

\begin{abstract}
10.1109/iros.2010.5649207
2010 IEEE/RSJ International Conference on Intelligent Robots and Systems IEEE
\end{abstract}

All content in PEARL is protected by copyright law. Author manuscripts are made available in accordance with publisher policies. Please cite only the published version using the details provided on the item record or document. In the absence of an open licence (e.g. Creative Commons), permissions for further reuse of content should be sought from the publisher or author. 


\title{
Workspace Comparisons of Setup Configurations for Human-Robot Interaction
}

\author{
Franziska Zacharias, Ian S. Howard, Thomas Hulin, and Gerd Hirzinger
}

\begin{abstract}
In virtual assembly verification or remote maintenance tasks, bimanual haptic interfaces play a crucial role in successful task completion. This paper proposes a method for objectively comparing how well a haptic interface covers the reachable workspace of human arms. Two system configurations are analyzed for a recently introduced haptic device that is based on two DLR-KUKA light weight robots: the standard configuration, where the device is opposite the human operator, and the ergonomic configuration, where the haptic device is mounted behind the human operator. The human operator directly controls the robotic arms using handles. The analysis is performed using a representation of the robot arm workspace. The merits of restricting the comparisons to the most significant regions of the human workspace are discussed. Using this method, a greater workspace correspondence for the ergonomic configuration was shown.
\end{abstract}

\section{INTRODUCTION}

All systems designed to be operated by a human have to provide a human-machine interface for their users. Systems built for applications like virtual reality simulations or teleoperation which require operators to act intuitively with the system, need interfaces that display the virtual environment as realistically as possible. For virtual assembly verification or remote maintenance tasks with haptic feedback, the quality of a haptic interface is crucial for successful task completion. The interface should allow flexible interaction with the operator. In the bimanual human-robot interface [1] analyzed in this paper, the operator uses both hands to directly control the robotic arms by attached handles (Fig. 1). The robot can be moved around and provides haptic feedback from a virtual environment to the user. Given there are two robotic arms in such a setup, the question is how to attach them to a base to allow for the best interaction with the operator. On the one hand, various setups can be evaluated using a number of subjects. Alternatively, they can be analyzed using functional criteria. In this paper, a method is presented to objectively evaluate the quality of a setup. The workspaces of the robot and the operator's arm are represented using the workspace model introduced by Zacharias et al. [2]. Additional statistical data obtained with a portable tracking system allows to restrict the workspace comparison to the most significant regions.

Franziska Zacharias, Thomas Hulin, and Gerd Hirzinger are affiliated with the Institute of Robotics and Mechatronics, German Aerospace Center (DLR), Wessling, Germany, franziska.zachariasedlr.de

Ian Howard is affiliated with the Department of Engineering, University of Cambridge, Cambridge, United Kingdom.
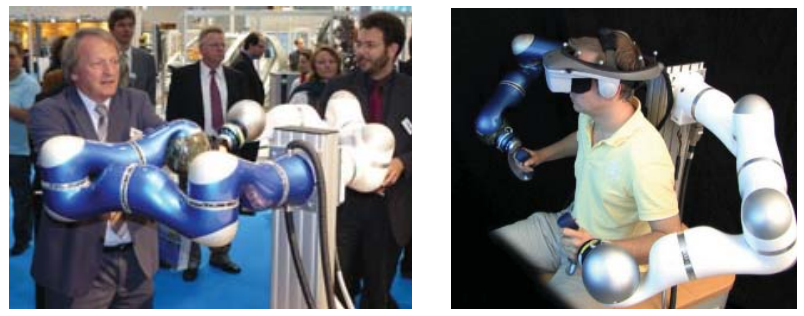

Fig. 1. Two-robot system providing bimanual haptic feedback. (left) Standard configuration, (right) Ergonomic configuration of the bimanual haptic interface.

\section{Problem statement}

Different configurations of the bimanual haptic device used in this work are possible. Two fundamentally different setups can be distinguished. Either the human interacts headon with the system (Fig. 1 left) in scenario 1, or the human is enclosed by the system (Fig. 1 right) in scenario 2. To evaluate the quality of one scenario with respect to the other, the most important question is how and what aspects can be compared. The first method of comparison that comes to mind would be to determine which scenario is easier to use. Psychological questionnaires and test tasks involving experimental trials carried out by subjects are typically used to perform this kind of evaluation. However this method is time consuming, requires a significant number of subjects and provides no quantitative measures of performance.

On the other hand an objective comparison should answer the following question: How well is the bimanual haptic interface able to mimic typical bimanual manipulation movements? This would enable a broad range of applications for the haptic interface. This paper concentrates on a method realizing this impartial comparison. A key point of the interaction is that the bimanual interface should not hinder the operator's desired movements during task execution. When the operator wants to reach a position in a certain orientation, e.g. to install a car battery or turn a screw, the interface should accommodate that movement. This requires that the robotic interface should cover the workspace of the human arm as well as possible. For the system configurations shown in Fig. 1, a complete coverage is not possible due to collisions between the robot arms and the human. For the systems discussed here therefore the workspace overlap has to be quantified. The simplest method of comparison would be to compute the maximum volume that can be swept by the robotic arm or the operator's arm e.g. using methods such as presented in [3] or [4]. One could then determine how 

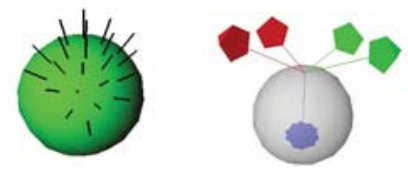

Fig. 2. An example subregion. (left) A set of reachable frames visualized as lines on a sphere. (right) Frames that correspond to a point on the sphere.

much of the volume for the operator's arm is covered by the robotic arm. However, a swept volume only specifies the positions that can be reached. No information regarding orientations is represented. This method is inadequate for cases where, for a set of positions, only certain orientations are of interest or where a certain region should be reachable in a versatile manner. Therefore this paper proposes to represent the workspace using the model described in section III. Position as well as orientation information is encapsulated. The model is computed for the kinematics defined in section IV. Section V details the comparison of the workspaces. In section VI scenario 1 and scenario 2 are evaluated. The merits of restricting the comparison to the most significant regions of the human workspace are also discussed.

\section{REPRESENTATION OF KINEMATIC CAPABILITIES}

In the reachable workspace volume, positions can be reached in at least one orientation. In the dexterous workspace volume positions can be reached in all orientations [5]. In practice, orientations are important but seldom all are needed. Therefore the model in [2] represents how close subregions are to belonging to the dexterous workspace and which orientations are possible. The proposed model, called the reachability map of the robot arm, represents its discretized workspace. For each subregion the reachability of a set of representative frames is examined and recorded. A frame here specifies the position and orientation of the end-effector coordinate system with respect to the reference system of the subregion. Fig. 2 (left) shows a set of reachable frames visualized as lines on a sphere. A line shows from which direction the specific point on the sphere is reachable. Regions on the sphere containing no lines are not reachable. Fig. 2 (right) shows two exemplar frames corresponding to one of the points on the sphere. The aggregation of these examined subregions of the workspace builds the reachability map. It is computed for each robot arm offline. The map is build once and can then be consulted to determine which regions are reachable from which directions. Fig. 3 shows a visualization of the reachability map for the right robot arm of the scenario 1 . The color encodes the reachability index. This index measures how well a region is reachable, i.e. what percentage of the tested frames is reachable for this region. Red denotes the minimum value and blue denotes the maximum value. Although this is a directionless index, that is not informative from which direction the region can be reached, it shows from how many directions the region can be reached and therefore gives an impression of the capabilities of the robot arm in its workspace. The data
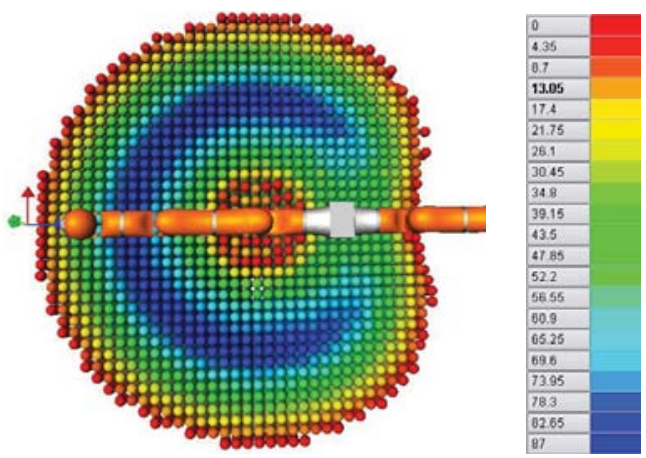

Fig. 3. Visualization of the reachability map for a robot arm. The map was cut in half for better visualization.

\begin{tabular}{c|c|c|c|c|c|c}
$\mathrm{i}$ & $\alpha_{i-1}$ & $a_{i-1}$ & $d_{i}$ & $\theta_{i}$ & $\mathrm{ll}$ & $\mathrm{ul}$ \\
\hline 0 & 0 & 0 & 0.31 & 0 & -170 & 170 \\
1 & 0 & 90 & 0 & 0 & -120 & 120 \\
2 & 0 & -90 & 0.4 & 0 & -170 & 170 \\
3 & 0 & -90 & 0 & 0 & -120 & 120 \\
4 & 0 & 90 & 0.39 & 0 & -170 & 170 \\
5 & 0 & 90 & 0 & 0 & -130 & 130 \\
6 & 0 & -90 & 0 & 0 & -170 & 170
\end{tabular}

TABLE I

DH PARAMETERS FOR THE ROBOT ARM.

indicating which of the individual frames is reachable is also available and is used in the analysis.

\section{KINEMATIC DESCRIPTIONS}

In this section the kinematics for the robot arm and the human arm is specified. They are used for the computation of the previously discussed workspace representation. Special emphasis is placed on the choice of the tool center point (TCP) i.e. the point of grasp contact used in this paper.

\section{A. Robot Arm Kinematics}

In both setups, the bimanual haptic interface is composed of two DLR-Kuka Light-Weight Robot arms. Each arm has seven degrees of freedom (DOF), mimicking the human arm kinematics and is therefore well suited for use in human robot interaction. Both arms have an identical kinematics and only differ in their attachment to the base. Tab. I describes the kinematics using Denavit-Hartenberg $(\mathrm{DH})$ parameters, as proposed by Craig [5]. The lower limits are shown in column $l l$ and the upper limits in column $u l$.

\section{B. Human Arm Kinematics}

This section details how the human arm kinematics is modeled. Two possibilities are discussed. The shoulder link is a spherical joint that is usually approximated by three serial axes. Tondu et al. [6] chose the first axis to point normal to the plane of the back of the human. In contrast, Abdel-Malek [3] chose the first axis to point outwards to the side of the human shoulder. Both versions are shown in Fig. 4 using coordinate systems. The coordinate axes are colorcoded with the $\mathrm{x}$-axis in red, the $\mathrm{y}$-axis in green and the $\mathrm{z}$-axis (=the rotation axis) in blue. One difference between 

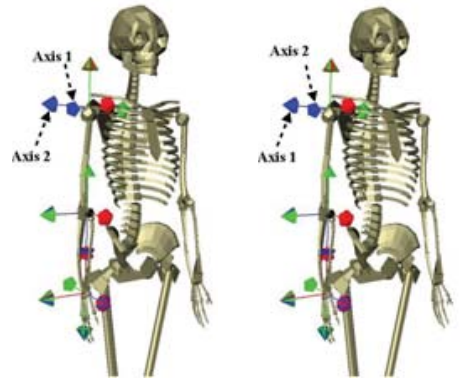

Fig. 4. Positions of the coordinate systems for the human arm kinematics. (left) Kinematics 1: First rotation axis points normal to the plane of human's back. (right) Kinematics 2: First axis points to the side.
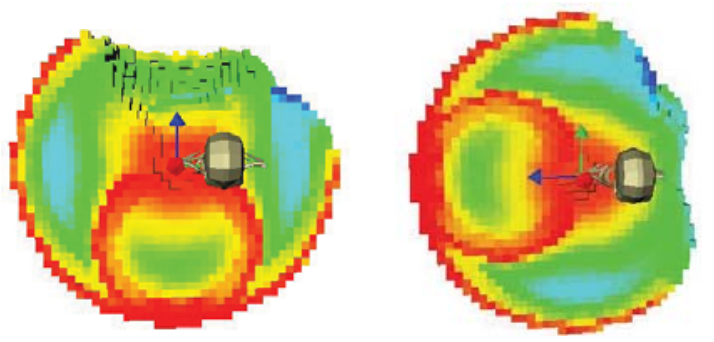

Fig. 5. Viewed from the top: (Left) Singularities for human arm kinematics 1. (right) Singularities for human arm kinematics 2 .

these two kinematics is the location of their singularities. Singularities can e.g. be detected using the volume of the manipulability ellipsoid [7]. In Fig. 5 bright red regions signify Cartesian regions where corresponding configurations could be singular, i.e. the volume of the manipulability ellipsoid is zero. In the first case, the Cartesian region where corresponding configurations might be singular lies to the front of the body. In the second case it lies on the right side of the right shoulder. To determine which kinematics better represents the human arm kinematics, the reachability map was computed for both versions. Fig. 6 shows the regions that received a Reachability-Index in the top $20 \%$ of the value range (blue) of the reachability map for kinematics 1 and 2 . It can be seen that whereas kinematics 2 conveniently turns the singularities out of the area where manipulation most commonly occurs, it also rotates the best reachable region out of that area. Best reachable region in terms of
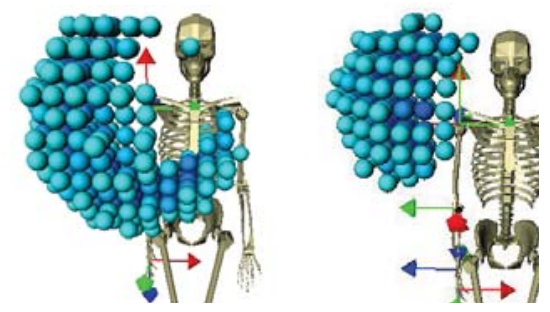

Fig. 6. Shows the regions that received a Reachability-Index in the top $20 \%$ of the value range (blue) for kinematics 1 (left) and 2 (right).

\begin{tabular}{c|c|c|c|c|c|c}
$\mathrm{i}$ & $\alpha_{i-1}$ & $a_{i-1}$ & $d_{i}$ & $\theta_{i}$ & $\mathrm{ll}$ & $\mathrm{ul}$ \\
\hline 0 & 0 & 0 & 0 & 0 & -30 & 180 \\
1 & 0 & 90 & 0 & -90 & -50 & 180 \\
2 & 0 & 90 & $d_{2}$ & 0 & -110 & 80 \\
3 & 0 & -90 & 0 & 0 & 0 & 145 \\
4 & 0 & 90 & $d_{4}$ & 0 & -85 & 90 \\
5 & 0 & -90 & 0 & 90 & -45 & 15 \\
6 & 0 & 90 & 0 & 90 & -85 & 85
\end{tabular}

TABLE II

DH PARAMETERS AND LINK LIMITS FOR THE HUMAN ARM.

the reachability index here means that the largest percentage of representative frames (compare Sec. III) is reached. This is clearly undesirable especially since Howard et al. [8] showed that the region the human hand moves in during every day tasks mostly lies to the front of the body. It is plausible to assume that the human arm's abilities are best in this region. This analysis also shows why the workspace representation summarized in Sec. III was used instead of a manipulability measure based workspace representation. For the wrist axes the same analysis can be performed. However due to the resolution of $0.05 \mathrm{~m}$ of the reachability map, this will not introduce significant changes with respect to the general location of the best reachable region. Therefore this analysis was not performed. Summing up, for the analysis in this paper kinematics 1 was chosen. It corresponds to the sequence and orientation of the link coordinate systems as detailed in Tondu et al. [6]. The kinematics (Tab. II) are expressed using the Denavit-Hartenberg (DH) parameters as given by Craig [5]. The link limits were set as proposed by Kapandji [9]. The length of the limbs was computed from real data using the method proposed by Howard et al. [8].

\section{The Selection of the Tool Center Point}

The selection of the tool center point (TCP) is essential for the comparison of the workspaces. The TCP is defined as the coordinate system that is reached by a homogeneous transformation multiplied onto the last link's coordinate system. It is the reference point for manipulation tasks. The importance of its selection can be easily recognized when considering the task of hitting a nail with a hammer. The structure of the workspace of the arm with a tool attached is considerably different from the workspace of the arm alone. The reason for this is the different location of the point of manipulation, i.e. the TCP. The selection of the TCP is reflected in the structure of the reachability map. Since the workspace maps are to be compared, the contact points between the two systems have to match.

For both scenarios the TCP for the human operator was chosen so that it lies at that point in the palm where the handle (compare with Fig. 1) is grasped. The TCP of the robot arm differs between the two setups. The goal of the workspace comparison is to evaluate how well the robot arm is able to achieve the positions and orientations requested by the human operator. Therefore the TCP for the robot arm has to be chosen so that it is identical with that of the human operator at the requested hand pose (Fig.7 a). The robot 


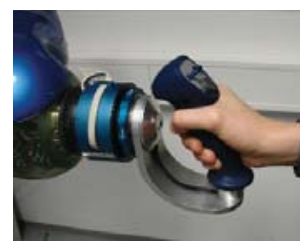

(a)

(c)

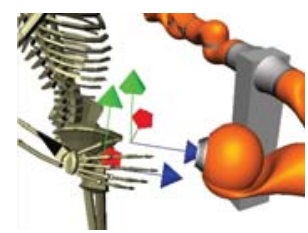

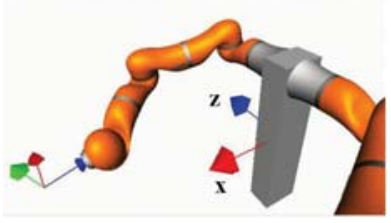

(b)

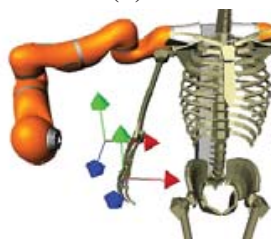

(d)
Fig. 7. (a) The hand grasping the handle attached to the robot arm in scenario 1. (b) The world coordinates system. TCP for scenario 1 (c) and scenario $2(\mathrm{~d})$.
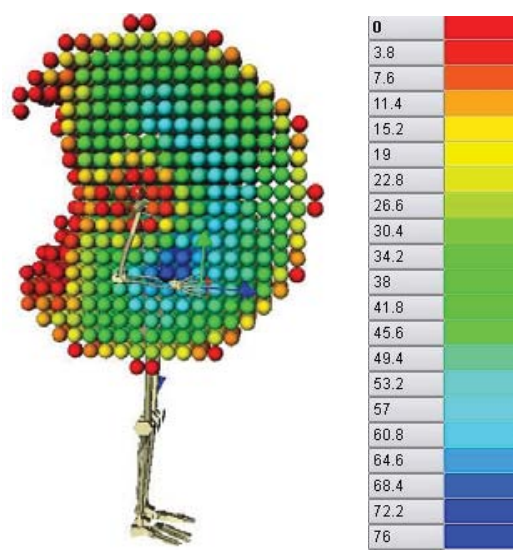

Fig. 8. Visualization of the reachability map for the human arm. The map was cut along in half along the xz-plane for better visualization.

arm TCPs are shown as coordinate systems in Fig. 7 (c) for scenario 1 and in Fig. 7 (d) for scenario 2. The human arm TCP is visualized in the human's palm.

\section{WORKSPACE COMPARISON}

Using the TCPs defined in the last section, the reachability maps are computed for the human arm and the robot arm. A visualization of a map for the robot arm is shown in Fig. 3 and the map for the human arm is shown in Fig. 8. The reachability map for the right arm of the human matches the intuitive expectations. The space where the most frames are reachable, lies in front of the body and can be reached with the elbow link bent at 90 degrees.

In the following analysis it is assumed that the pose of the human arm base and the pose of the robot arm base are known. To compare the workspaces each frame that is labeled reachable in the human arm's reachability map is transformed into the respective robot arm's coordinate system. It is then mapped to the best fitting frame in the robot arm's reachability map. Since the reachability map represents a discrete quantization of the robot's workspace, the frames are not identical. By best fitting, the frame that is closest in position and orientation is meant. The mapping process is identical to the one specified in [10]. First the position is mapped to one of the discretized subregions. Next the orientation of the z-axis is mapped. Finally the orientation of the $\mathrm{x}$-axis is mapped. As a result we get the frame of the subregion that fits best. The percentage of reachable frames for the human that can also be reached by the robot quantifies the overlap between the workspaces of the human and the robot arm. The method is summarized in Algorithm 1. The robotic system itself is stationary. However the position of

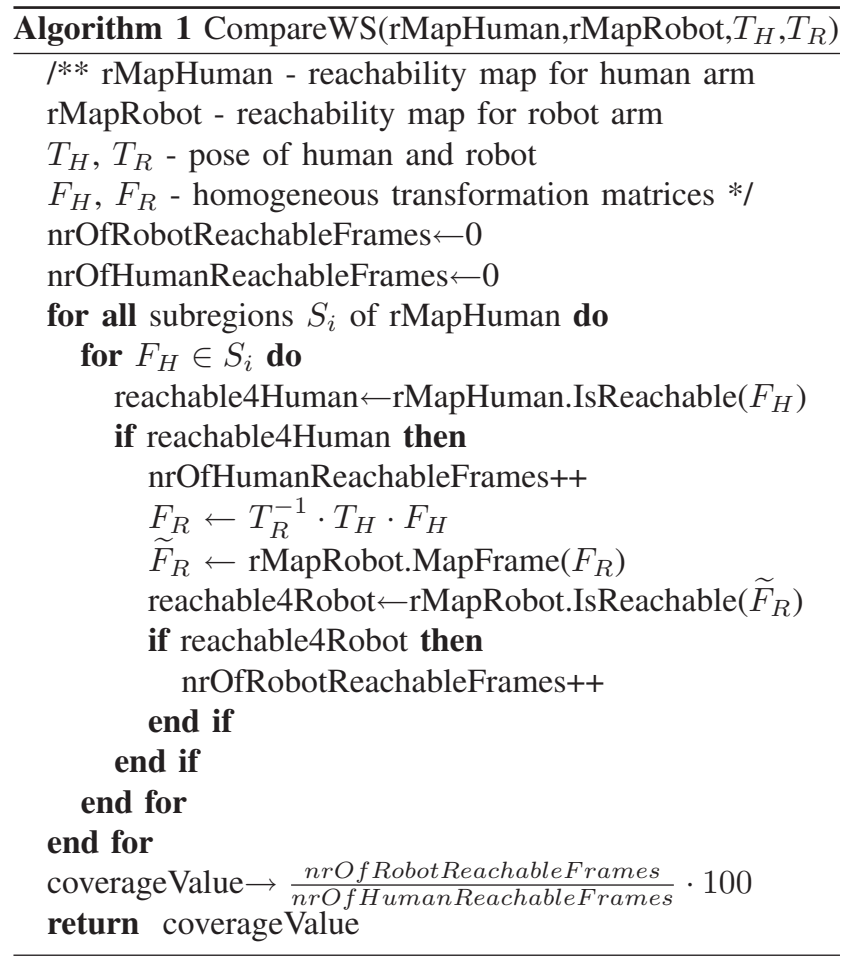

the human $T_{H}$ can vary with respect to the position of the robot system $T_{R}$. The comparison is performed for a discrete set of poses of the human with respect to the robotic system. Both arms of the human should be able to interact with their corresponding robot arms equally well. Therefore the human shoulders are parallel to the robot system and the orientation of the human with respect to the robot is not changed. For the same reason the human is centered with respect to the robot base.

\section{Evaluation}

In this section the proposed method will be evaluated. First the comparison was performed across the whole reachable workspace of the human arm. Recently, experiments as reported by Howard et al. [8] have shown that location of the wrists during daily tasks are concentrated in a subregion of the reachable workspace. The influence on setup comparisons made using this subregion is also demonstrated. Scenario 1 (Fig. 9 left) and scenario 2 (Fig. 9 right) were evaluated using algorithm 1. The main difference between the two scenarios 

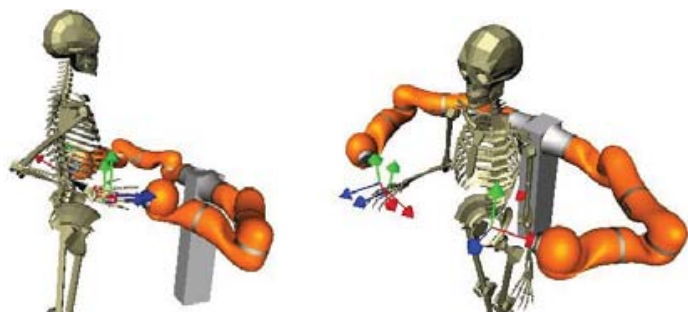

Fig. 9. Human interacting in scenario 1 (left) and in scenario 2 (right).
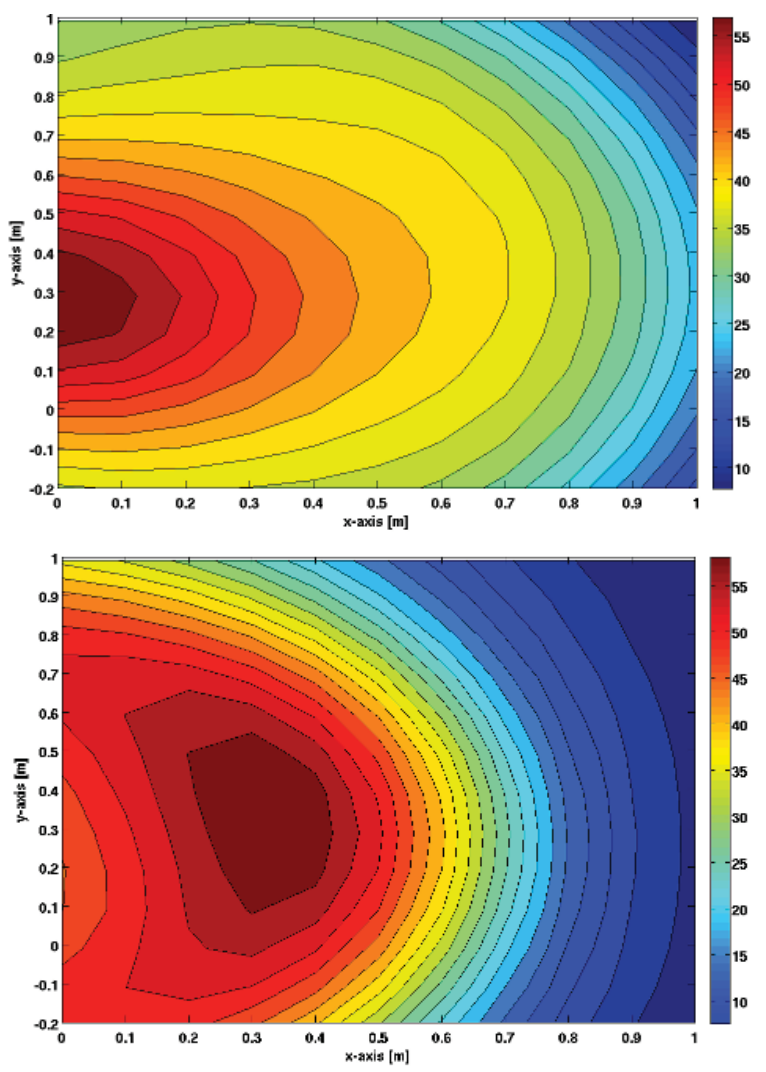

Fig. 10. Coverage values for different positions of the human shoulder with respect to the robot base. (top) for scenario 1 and (bottom) for scenario 2 .

is the pose of the human operator's shoulder with respect to the robot arms and the attachment of the handle to the end of the robot arm. Between the two scenarios, the handle was rotated by 90 degrees.

\section{A. Comparison made using the whole workspace}

In scenario 1 the right arm of the human interacts with the left arm of the two-arm robotic system. In scenario 2 the right arm of the human interacts with the right arm of the robotic system. The base of the two-arm robotic system is positioned at the origin of the global reference coordinate system. The human's shoulder is repositioned in the xy-plane with respect to the robots. The extrinsic coordinate system is displayed in Fig. 7 (b). It is placed in the center of the base block below the robotic arms, i.e. $0.29 \mathrm{~m}$ below the robot arms' bases. For each position of the human's shoulder is plotted how well the robot arm covers the human arm's workspace. The results for scenario 1 (Fig. 10 (top)) are compared with the results for scenario 2 (Fig. 10 (bottom)). Although the maximum coverage value does not differ significantly between the two scenarios, its peak location does. In scenario 1 at maximum $59 \%$ of the human workspace could be covered by the robot arm. In scenario 2, 63\% coverage was obtained. So far, collisions between the human and the robot were not taken into account. In practice such collisions will affect the results of the analysis. In scenario 1 the maximum coverage occurs when the human shoulder is placed at location $(x, y)=(0 m, 0.29 m)$. This is to be expected since the bases of the robot arm and the human arm coincide and the coverage is optimal. However at this location both arms are permanently in collision. Therefore the human operator cannot be positioned at the location of this maximum. The x-component of the human's shoulder position has to be greater than $x=0.5 \mathrm{~m}$ to avoid collisions with the robot and still be able to interact with the robot arms as shown in Fig. 9. Thus, the maximum coverage decreases to $\approx 43 \%$ at $(0 \mathrm{~m}, 0.5 \mathrm{~m})$. In scenario 2 the maximum coverage occurs for the human shoulder at location $(0.35 \mathrm{~m}, 0.35 \mathrm{~m})$. Fig. 9 (right) shows the human assuming this position. No collisions occur. In fact any position with an x-component $x \geq 0.2 m$ is feasible, i.e. no collisions occur and the human can operate the system as shown in Fig. 9 (right). The $z$ component is fixed in these analyzes. It was chosen so that the human is centered between both robotic arms.

\section{B. Comparison using a restricted human workspace}

The previous section evaluated the coverage of the entire reachable workspace of the human arm by the robotic arm. However, as recent research shows [8] only a limited portion of the human arm's workspace is used in every day life. When analyzing virtual manufacturing scenarios, additional ergonomics aspects could also be considered. For example, especially situations where a person manipulates above the head should rarely occur in manufacturing tasks. The postures, the human would have to assume, receive bad ergonomic scores [11]. If they were executed often, they would result in damage to the musculoskeletal system of the person involved. For these reasons the comparison of the workspaces is restricted to that region primarily used by the human. In [8] data was collected from subjects who wore a portable motion tracking system to record their arm movements as they went about their daily life. No specific instructions were given and the system allowed the subject to engage spontaneously in normal every day tasks. The results show that the majority of wrist positions fall within a region close to the body and also mostly to the front of the body. Fig. 11 shows two views of the dataset for one subject. In the following, the comparison is restricted to that part of the human arm's workspace that overlaps with the natural movement data. Thus the robot arm only has to cover this restricted workspace. The position of the palm was extrapolated from the wrist position. Using the restricted workspace, comparison results are shown in Fig. 12 (top) for 

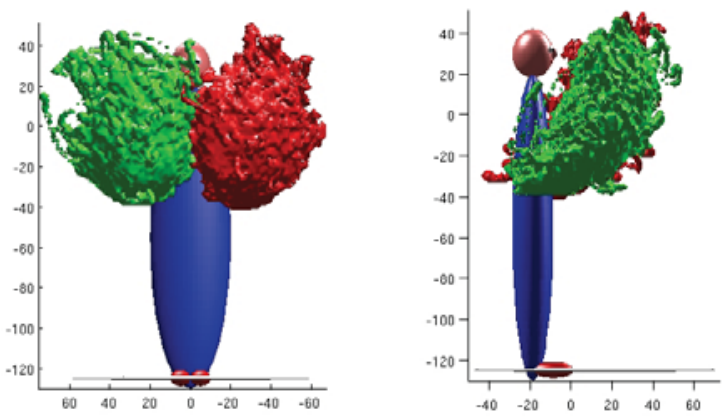

Fig. 11. Distribution of the wrist position recorded with a portable tracking system as the subject went about his daily life.
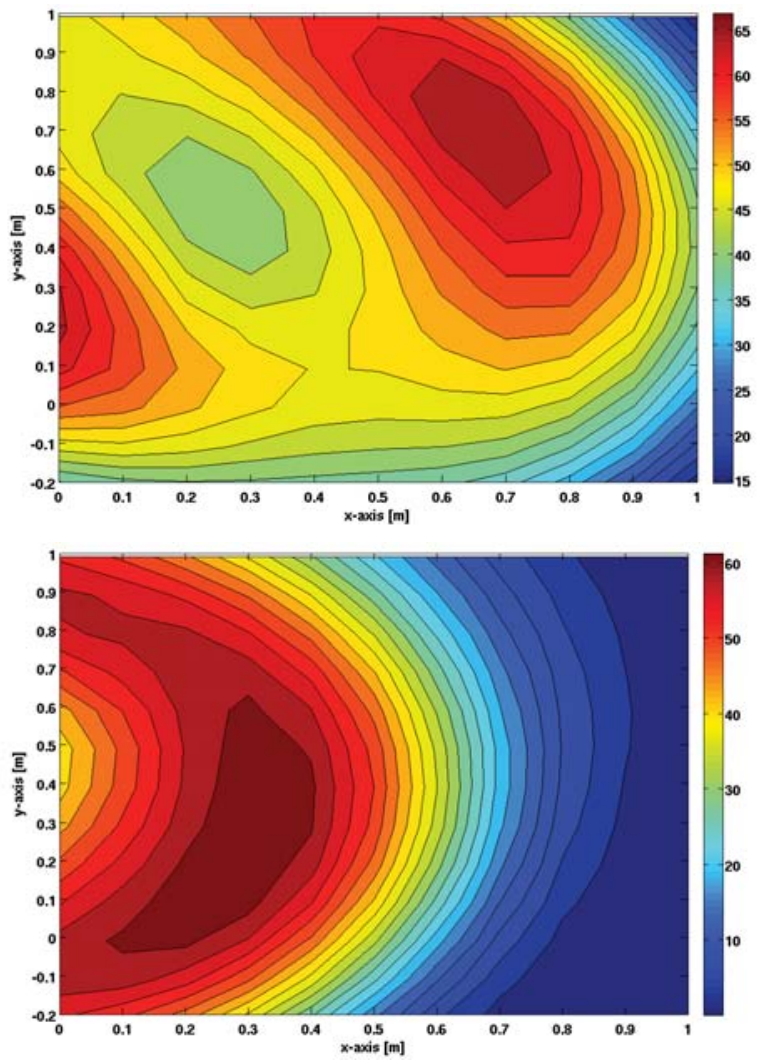

Fig. 12. Comparison results for the human shoulder placed in the xy-plane. The human interacting with (Top) scenario 1 and (Bottom) scenario 2.

scenario 1 and in Fig. 12 (bottom) for scenario 2. It can be seen that the height of the maximum (region in dark red) does not differ significantly. In scenario 1 the maximum coverage is $66.8 \%$ and in scenario 2 the maximum coverage is $64.4 \%$. The Cartesian region where the human can be positioned to reach these maxima is collision free in both cases. According to the results using the restricted natural workspace, it is recommended to implement scenario 2 since the area of the region where the maximum coverage occurs is greater. Furthermore its extension in the y-direction is larger. This signifies that the shoulder height of the operator is allowed to vary over a greater range without the need to reposition the user. Thus different operators can more easily achieve a good performance with the system if they are located at a fixed position with respect to the robotic system. Additionally, in scenario 2 the danger of the robot hindering the human by being in the way is reduced.

\section{CONCLUSION}

A method is presented to objectively evaluate robotic setup configurations in which a human operator has to directly interact with robotic arms. The method was applied to two scenarios. The choice of data for the comparison was discussed and its effect on the evaluation was demonstrated. As shown, the coverage value varies with the relative position of human and robots. The placements of the human shoulder can be identified for which good performance is achieved. In future work, the proposed method can therefore be used as a quality criterion for optimizing human-robot interaction setups. In conclusion, the ergonomic scenario 2 is more attractive, especially since the location of the maximum coverage is approximately the same in the comparison across the whole workspace and in the comparison using the workspace filtered by the natural human movement data. Therefore, this system setup has greater potential for more general tasks.

\section{ACKNOWLEDGMENTS}

The authors would like to thank LAAS-CNRS for providing the graphics models of the virtual human and Daniel Wolpert for allowing the use of the natural movement dataset. The research has been partially funded by the EC Seventh Framework Programme (FP7) under grant agreement no. 216239 as part of the IP DEXMART. Ian Howard was supported by the European Project SENSOPAC (IST-2005028056).

\section{REFERENCES}

[1] T. Hulin, M. Sagardia, J. Artigas, S. Schaetzle, P. Kremer, and C. Preusche, "Human-scale bimanual haptic interface," in Proc. 5th International Conference on Enactive Interfaces, 2008.

[2] F. Zacharias, C. Borst, and G. Hirzinger, "Capturing robot workspace structure: Representing robot capabilities," in Proc. IEEE Int. Conf. on Intelligent Robots and Systems (IROS), 2007, pp. 3229-3236.

[3] K. Abdel-Malek, J. Yang, R. Brand, and E. Tanbour, "Towards understanding the workspace of human limbs," Journal of Ergonomics, vol. 47, pp. 1386-1405, 2004.

[4] I. Rodriquez, M. Peinado, R. Boulic, and D. Meziat, "Bringing the human arm reachable space to a virtual environment for its analysis," in Proc. Int. Conf. on Multimedia and Expo, 2003, pp. 229-232.

[5] J. Craig, Introduction to Robotics: Mechanics and Control. AddisonWesley, 1989.

[6] B. Tondu, S. Ippolito, and J. Guiochet, "A seven-degrees-of-freedom robot-arm driven by pneumatic artificial muscles for humanoid robots," J. Robotics Research, vol. 24, no. 4, pp. 257-274, 2005.

[7] T. Yoshikawa, Foundations of Robotics: Analysis and Control. Cambridge, Massachusetts: MIT Press, 1990.

[8] I. Howard, J. Ingram, K. Körding, and D. Wolpert, "Statistics of natural movements are reflected in motor errors," J. Neurophysiol., vol. 102, no. 3, pp. 1902-1910, 2009.

[9] I. Kapandji, The Physiology of the Joints - Upper Limb. Churchill Livingstone, 1982, vol. 1

[10] F. Zacharias, W. Sepp, C. Borst, and G. Hirzinger, "Using a model of the reachable workspace to position mobile manipulators for 3-d trajectories," in Proc. IEEE Int. Conf. on Humanoid Robots, 2009.

[11] L. McAtamney and E. Corlett, "Rula: a survey method for the investigation of work-related upper limb disorders," Applied Ergonomics, vol. 24 , pp. 91-99, 1993. 